\title{
MicroRNAs as biomarkers for prostate cancer prognosis: a systematic review and a systematic reanalysis of public data
}

\author{
Sharmila Rana ${ }^{1}$, Gabriel N. Valbuena ${ }^{2}$, Ed Curry ${ }^{3}$, Charlotte L. Bevan $\mathbb{D}^{1 凶}$ and Hector C. Keun (D) ${ }^{1 凶}$ \\ (c) The Author(s) 2022
}

BACKGROUND: Reliable prognostic biomarkers to distinguish indolent from aggressive prostate cancer (PCa) are lacking. Many studies investigated microRNAs (miRs) as PCa prognostic biomarkers, often reporting inconsistent findings. We present a systematic review of these; also systematic reanalysis of public miR-profile datasets to identify tissue-derived miRs prognostic of biochemical recurrence $(B C R)$ in patients undergoing radical prostatectomy.

METHODS: Independent PubMed searches were performed for relevant articles from January 2007 to December 2019 . For the review, 128 studies were included. Pooled-hazard-ratios (HRs) for miRs in multiple studies were calculated using a random-effects model (REM). For the reanalysis, five studies were included and Cox proportional-hazard models, testing miR association with BCR, performed for miRs profiled in all.

RESULTS: Systematic review identified 120 miRs as prognostic. Five (let-7b-5p, miR-145-5p, miR152-3p, miR-195-5p, miR-224-5p) were consistently associated with progression in multiple cohorts/studies. In the reanalysis, ten (let-7a-5p, miR-148a-3p, miR-203a$3 p$, miR-26b-5p, miR30a-3p, miR-30c-5p, miR-30e-3p, miR-374a-5p, miR-425-3p, miR-582-5p) were significantly prognostic of BCR. Of these, miR-148a-3p ( $\mathrm{HR}=0.80 / 95 \% \mathrm{Cl}=0.68-0.94)$ and $\operatorname{miR}-582-5 p(\mathrm{HR}=0.73 / 95 \% \mathrm{Cl}=0.61-0.87)$ were also reported in prior publication(s) in the review.

CONCLUSIONS: Fifteen miRs were consistently associated with disease progression in multiple publications or datasets. Further research into their biological roles is warranted to support investigations into their performance as prognostic PCa biomarkers.

British Journal of Cancer (2022) 126:502-513; https://doi.org/10.1038/s41416-021-01677-3

\section{BACKGROUND}

Prostate cancer $(\mathrm{PCa})$ is the most common cancer and second most lethal cancer in men in the UK over 40,000 cases were diagnosed and around 11,700 deaths occurred every year between 2015 and 2017 [1]. It is a heterogeneous disease and can manifest as either a low-risk, indolent tumour localised to the prostate or a high-risk, aggressive tumour that eventually metastasises and proves lethal if untreated. As many as $42-66 \%$ of patients present with the indolent form of PCa [2,3]. Over the last three decades, PCa incidence has rapidly increased while the mortality rate has remained relatively stable [1]. This rise in incidence is attributed to the widespread use of the prostate specific antigen (PSA) test in diagnosing PCa. However, this test is not specific and results in a high proportion of false positives as well as detection of indolent disease [2, 4]. This has led to overtreatment of patients without any benefit in overall survival, evidenced by the steady mortality rate, and has led to an increase in PCa disease burden. Additionally, $15-45 \%$ of patients treated with radical prostatectomy (RP), one of the first-line curative treatments for localised $\mathrm{PCa}$, experience biochemical recurrence (BCR) within 5-years [5-8]. Although BCR does not always equate to clinical recurrence, it is considered an initial event signifying disease progression and has shown to be associated with increased risk of PCa metastasis and cancer-specific mortality [711]. These problems highlight the importance of reliable and accurate identification of aggressive disease as distinct from indolent disease in order to limit over-treatment and provide appropriate treatment strategies for the management of PCa.

Current prognostic markers used for disease management decisions are based on risk stratification systems which incorporate clinicopathological variables Gleason score, pathological tumour stage and serum PSA at diagnosis [12, 13]. Although these variables are good indicators of disease severity and correlate with patient survival, their measurements are subject to sampling and random errors as the biopsies may miss tumours, resulting in a high proportion of misdiagnoses. More than $30 \%$ transrectal ultrasound biopsies are false negatives and higher than $45 \%$ of cancer patients have their Gleason scores underestimated $[14,15]$. In addition to the risk stratification systems, there are various promising multi-omic biomarker panels currently being explored for prognostication such as the OncotypeDX Genomic Prostate Score, Decipher and Prolaris tests [16-18]. These panelbased tests have been validated in large external cohorts and often reach an AUC higher than 0.7 [17-21]. However, these tests

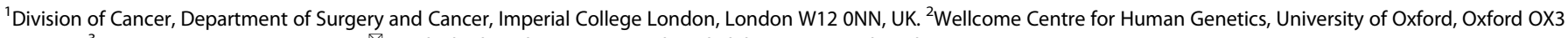
7BN, UK. ${ }^{3}$ GSK, Stevenage SG1 2NY, UK. ${ }^{\circledR}$ email: charlotte.bevan@imperial.ac.uk; h.keun@imperial.ac.uk 
are not widely available, for instance in the UK they are only commercially available in some private clinics, severely limiting accessibility both financially and geographically.

MiRs have been investigated for their potential to serve as alternative molecular markers for PCa. MiRs are small non-coding RNAs that negatively regulate gene expression at the posttranscriptional level. They do so by binding to complementary sequences in the $3^{\prime}$ UTR of target mRNAs via a preserved 'seed sequence' region, which then represses translation of the target mRNAs [22]. Due to their regulatory role, these molecules have been implicated in various developmental, cellular and physiological processes and their dysregulation has been associated with various diseases [22, 23]. Differential miR expression profiles between tumour and normal tissues have been observed in various cancers, including PCa [24-27]. Other advantages as biomarkers inlcude: miRs are abundantly and stably expressed invivo, detected in biofluids such as blood, urine and saliva, and are highly stable in storage [26-31].

The first extensive miR expression profiling in PCa cell lines, xenograft samples and clinical tumour samples was published in 2007 by Porkka and colleagues [32]. Since then, numerous studies have characterised miR expression profiles in PCa tissues and biofluids at various stages of the disease and examined their prognostic potential [27, 30, 33-42]. A major caveat to these studies is that they often report inconsistent results, possibly due to heterogeneity between studies, including differences in study designs, methodologies, and clinically diverse populations. Thus, there is no general consensus to date on the miRs that truly associate with disease progression and have the potential to be utilised as prognostic biomarkers for PCa. Attempts at metaanalyses to combine results from multiple studies and appraise the current miR biomarker landscape are limited to only a handful of publicly available datasets [43]. A systematic review, which does not require the disclosure of sensitive clinical datasets, may be more useful in examining the prognostic miR biomarker landscape in PCa and identifying consistent patterns across the studies. As yet, no such systematic review covering the topic of prognostic miR biomarkers in PCa has been published.

In this study, we aimed to review the relevant existing publications in the scientific literature to date and identify consistently reported miRs with potential as prognostic biomarkers in PCa. First, a systematic review was performed of studies that investigated the prognostic potential of individual miRs or miR panels in PCa. A comprehensive approach was taken in which any publications evaluating prognostic miRs were included, irrespective of methodological or clinical diversity. The review revealed a considerable number of publications that investigated the association of tumour tissue-derived miRs with BCR in patients who have undergone RP. The only meta-analysis of primary data addressing prognostic miRs in PCa was performed in 2017 [43]. To account for new public datasets after this, an updated reanalysis was performed on studies with publicly accessible global miR expression datasets. Based on the results of the systematic review, we redefined the aim to focus on identifying miRs that are prognostic of BCR in patients that have undergone RP. Here, only tissue-specific miRs were considered as the majority of publications $(\sim 88 \%)$ in the systematic review addressed tissuederived miRs.

\section{METHODS}

The systematic review and data reanalysis were conducted in accordance with the PRISMA guidelines [44].

\section{Methodology for systematic review}

Search strategy. A methodological search of electronic database PubMed was performed on 24th of January, 2020 for relevant studies published between January 2007 and December 2019. The keywords searched were 'prostate cancer microRNAs prognosis relapse outcome'. This search included both free words and MeSH terms, ensuring all publications with the keywords and related terms in their title or body were included in the search result. The MeSH terms associated with the keywords were: ('micrornas'[MeSH Terms] OR 'micrornas'[All Fields] OR 'mirnas'[All Fields] OR 'miRs'[All fields] OR 'microrna'[All Fields] OR 'mirna'[All Fields] OR 'miR'[All fields]) AND ('prostatic neoplasms'[MeSH Terms] OR ('prostatic'[All Fields] AND 'neoplasms'[All Fields]) OR 'prostatic neoplasms'[All Fields] OR ('prostate'[All Fields] AND 'cancer'[All Fields]) OR 'prostate cancer'[All Fields]) AND ('prognosis'[MeSH Terms] OR 'prognosis'[All Fields] OR 'recurrence'[MeSH Terms] OR 'recurrence'[All Fields] OR 'relapse'[All Fields] OR 'mortality'[Subheading] OR 'mortality'[All Fields] OR 'survival'[All Fields] OR 'survival'[MeSH Terms] OR 'outcome'[All Fields]).

Study eligibility. Studies were selected according to the following criteria:

(i) the study measured expression of miRs in tissues or circulation of PCa patients (not xenograft or other animal models);

(ii) the study performed a survival analysis to examine the association of miRs with outcome: Cox PH regression model or Kaplan-Meier (KM) analysis, and appropriate test statistics such as hazard ratio (HR), 95\% confident intervals $(\mathrm{Cl})$ and log-rank $p$-values were reported in the main text or supplementary section.

Studies were excluded if:

(i) the study tested the prognostic role of miR host genes or target genes instead of the miR itself;

(ii) the study tested the prognostic role of $\mathrm{miR}$ in combination with non-miR markers such as clinical factors, genes or proteins;

(iii) the study was in a different language with no English translation available;

(iv) the study was a meta-analysis, review, comment, letter or duplicate publication.

Data extraction. The following data were extracted from each eligible study: PMID, surname of first author, year published, title, miR(s) investigated, sample size, sample type, detection method, outcome endpoint, endpoint definition, test type (Cox $\mathrm{PH} / \mathrm{KM})$, effect estimates (HR, 95\% Cl or log-rank $p$-value), Cox $\mathrm{PH}$ test type (univariate/multivariate), adjusted variables (if multivariate $\mathrm{Cox} \mathrm{PH}$ ). If the study performed both Cox $\mathrm{PH}$ model and $\mathrm{KM}$ analysis, only the results for $\mathrm{Cox} \mathrm{PH}$ model was extracted.

Statistical analysis. For the miRs that had multiple entries for the same endpoint and had their Cox PH test statistics reported, a meta-analysis was performed in order to calculate the summary effect size (pooled HR). For miR entries originating from the same study, a fixed-effects model (FEM) approach was employed. For miR entries from different studies, we hypothesised that due to biological and technological diversity, the true effect size varied across studies. Thus, a random-effects model (REM) approach was employed for these miRs. Low miR expression was set as the reference group, so for entries with high miR expression as the reference group, reciprocal of $\mathrm{HR}$ and $95 \% \mathrm{Cl}$ were calculated. Between study heterogeneity was assessed using Cochran's Q-test and Higgins $I^{2}$ statistic. Significance for the $\mathrm{Q}$-test was defined as $p<0.05$. Due to the very small number of studies considered in the meta-analysis, publication bias was not assessed. The meta-analysis and heterogeneity tests were performed in statistical software R using package metafor (version 2.4.0) [45].

MiR annotation. As the search spanned more than a decade, the miR annotation was outdated in many of the studies. For such cases, the article was screened in order to obtain strand information for the miR of interest. If strand information was not stated in the article, the miR was assumed to be the dominant strand. The miR name was then cross-referenced with its entry in the miRBase database, which contains an archive of miR annotations and sequences for all species and updated to the most recent version (version 22) [46]. MiR names were left unchanged if the dominant/ passenger strand in miRBase was not specified.

Methodology for systematic reanalysis of public miR datasets Search strategy. A methodological search of electronic database PubMed was performed on 23rd of April, 2020 in order to identify relevant studies published between January 2007 and December 2019. The keywords 
searched were 'prostate cancer relapse microRNA expression'. The MeSH terms associated with the keywords were: ('prostatic neoplasms' $[\mathrm{MeSH}$ Terms] OR ('prostatic'[All Fields] AND 'neoplasms'[All Fields]) OR 'prostatic neoplasms'[All Fields] OR ('prostate'[All Fields] AND 'cancer'[All Fields]) OR 'prostate cancer'[All Fields]) AND ('recurrence'[MeSH Terms] OR 'recurrence'[All Fields] OR 'relapse'[All Fields]) AND ('micrornas'[MeSH Terms] OR 'micrornas'[All Fields] OR 'mirna'[All Fields]) AND ('gene expression'[MeSH Terms] OR ('gene'[All Fields] AND 'expression'[All Fields]) OR 'gene expression'[All Fields] OR 'expression'[All Fields]).

Study eligibility. Studies were selected according to the following criteria:

(i) the study measured miR expression in tissues of PCa patients who underwent RP and no other curative therapy (no studies with miRs profiled in circulation);

(ii) the study generated global miR expression profiling dataset which was available in public data repositories;

(iii) the study contained follow-up data, i.e. BCR status of patients and time to $B C R$.

Studies were excluded if:

(i) the study was in a different language with no English translation available;

(ii) the study was a meta-analysis, review, comment, letter, or duplicate publication.

For studies with publicly accessible expression datasets and insufficient follow-up information, corresponding authors were directly contacted for additional clinical information. Studies that examined miR expression profile without generating novel data were also included in order to examine if the datasets they used were suitable for our reanalysis.

Data extraction and normalisation. Five studies, which included six datasets, were eligible for the data reanalysis (Table 1). For the TCGAPRAD dataset, access to raw miR-sequencing data was granted through the NIH database of Genotypes and Phenotypes, and the raw miRsequencing data and associated clinical data were downloaded from the Genomic Data Commons data portal via the data transfer tool and Bioconductor package TCGAbiolinks (version 2.12.6) [47-49]. Raw miR expression data was normalised using the trimmed mean of $M$-values method using the edgeR package (version 3.26.8) [50]. MiRs were then filtered to include only those with normalised read counts $\geq 1$ counts per million in at least $80 \%$ of samples, which left 328 miRs. For the rest of the datasets, normalised miR expression data and associated clinical data were obtained from the NCBI Gene Expression Omnibus database [51]. For GSE21036, clinical information was supplemented with clinical data obtained from the data repository in the MSKCC computational biology centre website [https://cbio.mskcc.org/cancergenomics/prostate/data/]. For GSE26245 and GSE26247, clinical information was supplemented with clinical data provided in the supplementary section of their corresponding paper [52]. For GSE46738 and GSE88958, the corresponding authors directly provided follow-up data (Leite K., written communication, 27 June 2018; Ozen M., written communication, 18 January 2019). The normalised datasets were standardised according to $z$-score transformation. MiR annotation in each dataset was also updated to miRBase version 22 using package miRBaseConverter (version 1.8.0) [53].

Statistical analyses. Serum PSA at diagnosis, Gleason score sum and clinical tumour stage were available in five of the six datasets. Only GSE88958 did not contain tumour stage information. To account for this, firstly, a univariate Cox PH analysis was performed in each of the six datasets, where the only predictor being tested for association with disease relapse was miR expression. Secondly, a multivariate $\mathrm{Cox} \mathrm{PH}$ analysis was performed in each of the five datasets with all three clinicopathological features available. Here, the Cox PH model included miR expression as the main predictor with PSA, Gleason score sum and tumour stage as confounders. Cox $\mathrm{PH}$ regressions were performed using $\mathrm{R}$ package survival (version 3.1.12) [54]. Analysis of variance (ANOVA), Kruskal-Wallis (KW) and Chi-squared $\left(X^{2}\right)$ tests were also performed to test whether the distribution of the clinical variables differed between the datasets.

Following univariate/multivariate Cox $\mathrm{PH}$ analysis, a REM meta-analysis was performed to calculate the pooled HR of the miRs across the studies. The meta-analysis was performed only for miRs that were present in all the

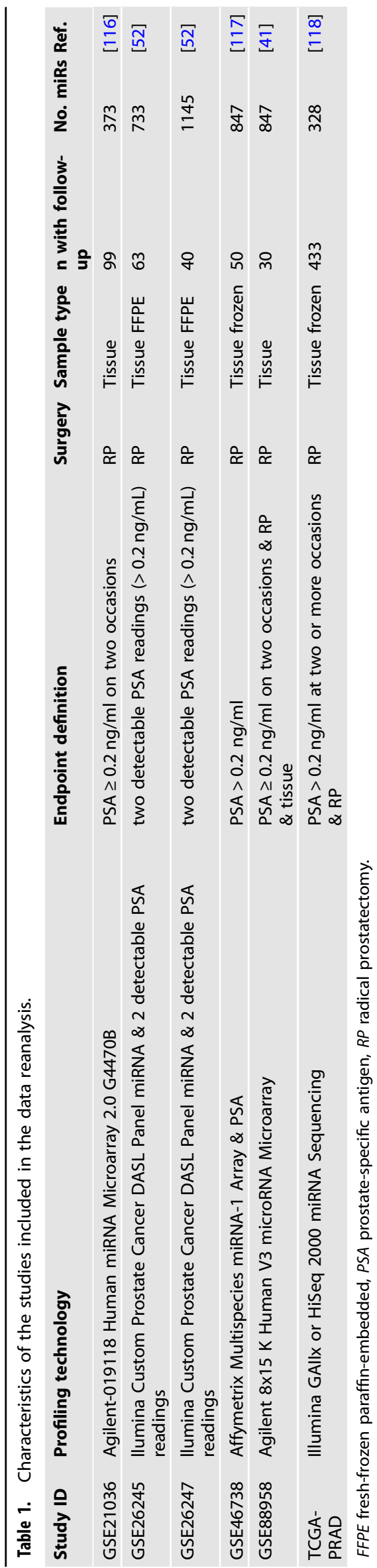


datasets. Subsequently, a total of 162 and 164 miRs were evaluated in the univariate and multivariate analyses, respectively. The significance threshold was set at $p$-value $<0.05$. As very few studies were included in this meta-analysis, publication bias was not assessed.

\section{RESULTS \\ Prognostic miRs in localised prostate cancer: a systematic review}

Study selection and characteristics. A total of 992 studies were retrieved from the initial literature search. Title and abstract screening removed 800 non-relevant studies such as metaanalyses, book chapters, reviews and other irrelevant publications. Full-text screening removed a further 64 studies for reasons such as inaccessibility of full text, insufficient reporting of results, no prognostic test performed and containing mistakes such as incorrect $\mathrm{Cls}$ or female PCa sample population. Ultimately, 128 studies were eligible and included 215 entries for individually prognostic miRs (containing 120 unique miRs) and 18 entries for miR signatures panels (containing 8 unique miR signatures). Workflow for study selection is detailed in Fig. 1 a.

The majority of miR biomarkers were detected using variations of the PCR technique ( $q P C R, R T-P C R, q R T-P C R)$. Less frequent detection methods were microarrays, (small-)RNAseq, NanoString, in situ hybridization, mass spectrometry and BeadChip based technologies. The review included biomarkers extracted from a variety of sources such as tissues $(n=204)$, blood (whole blood, peripheral blood, serum, plasma; $n=23)$, exosomes $(n=2)$, urine $(n=2)$ and cells (epithelial and stromal, fibroblast) $(n=2)$. The different clinical trial endpoints used by the studies in the review are listed in Table 2. The most common endpoint used as a surrogate for progression was biochemical recurrence-free survival (BPFS; 44.64\%), followed by overall survival (OS; 20.17\%). The remaining endpoints each accounted for less than $10 \%$ of the studies in the review. The study characteristics, statistical results, endpoint definitions and additional variables included in the survival analysis (if a multivariate $\mathrm{Cox} \mathrm{PH}$ was performed) are summarised in Supplementary Tables S1 and S2 for individually prognostic miRs and in Supplementary Tables S3 and S4 for prognostic miR signatures.

Individually prognostic miR biomarkers in PCa. We accumulated 215 entries reporting 120 unique prognostic miRs in $\mathrm{PCa}$ (Supplementary Table S1). 44 unique miRs had multiple entries in the review. These miRs were either evaluated against different endpoints/ cohorts in the same study or were evaluated more than once in separate studies. Of these, $36 \mathrm{miRs}$ had Cox $\mathrm{PH}$ output available. A REM meta-analysis was performed for the miRs evaluated against the same endpoint to determine their overall association (Supplementary Fig. S1). Seven miRs - let-7b-5p, miR$128 a-3 p$, miR-188-5p, miR-224-5p, miR-23a-3p, miR-23b-3p and $\mathrm{miR}-34 \mathrm{~b} / \mathrm{c}$ consistently and significantly associated with progression. High expression of miR-34b/c and miR-23a-3p associated with poor disease outcome, while for the remaining $5 \mathrm{miRs}$ high expression associated with better disease outcome. The Q-test for heterogeneity was not significant for these miRs (where metaanalysis was performed). Similarly, the $\mathrm{I}^{2}$ statistic ranged between 0.00 and $4.30 \%$, suggesting absence of statistical heterogeneity. A forest plot was also generated for the remaining miRs with single entries (Supplementary Fig. S2).

Considering the effect sizes of both $\mathrm{Cox} \mathrm{PH}$ and $\mathrm{KM}$ outputs for the 120 unique miRs, 57 miRs negatively associated with progression, 43 miRs positively associated with progression and 20 miRs had an inconsistent direction of association. Only four miRs, let-7b-5p, miR-152-3p, miR-195-5p and miR-224-5p, significantly and consistently associated with progression in multiple patient cohorts in the same study or in at least two independent studies. Additionally, miR-145-5p had an insignificant but

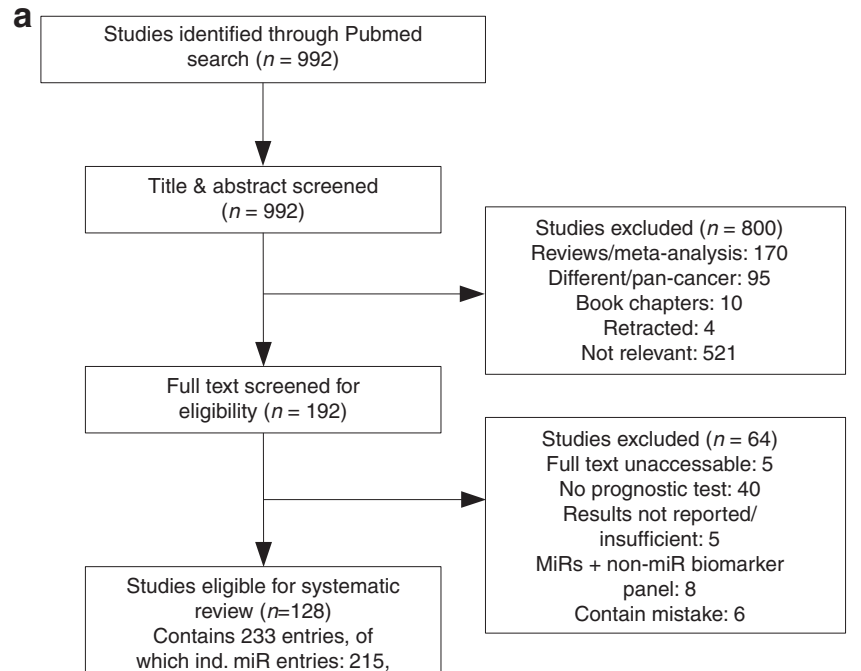

which ind miR entries: 215 miR signatures; 18

b

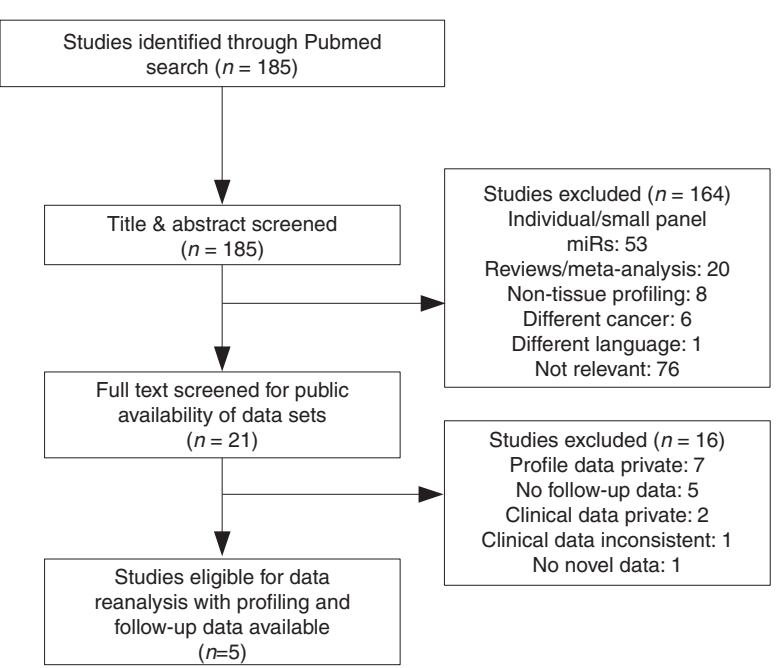

Fig. 1 Workflow for selecting eligible studies in the review. Workflow for the systematic review (a) and data reanalysis (b). Majority of the studies in the initial searches had themes on cancer, miRs and/or molecular biomarkers but did not directly address the primary subjects of the paper (i.e. PCa recurrence and miR biomarkers), so did not qualify for the analysis and were labelled as 'not relevant'.

consistent trend in association with progression in five independent studies. Low expression of all five miRs consistently associated with shorter time to disease progression (Table 3, Supplementary Fig. S1). These miRs are the strongest prognostic biomarker candidates for PCa based on current literature.

Prognostic miR signatures as biomarker panels in $P C a$. Eight miR signatures, comprised of 36 unique miRs, were reported as prognostic in eight independent studies (Supplementary Table S3). The majority of these studies performed independent clinical validations and/or have large sample sizes ( $\& 100)$, making their findings robust. Interestingly, only Feng et al. (2017) investigated a panel of miRs that were biologically related, in this case the miRs in the signature panel were all part of the miR-17/92 cluster [55]. The remaining studies grouped miRs into signature panels if they were significantly differentially expressed between recurrent and nonrecurrent cases or individually had significant predictive power to distinguish between recurrent and non-recurrent cases. 
Table 2. Progression endpoints considered in the systematic review.

\begin{tabular}{|c|c|c|}
\hline Endpoint & Abbreviation & $\begin{array}{l}\text { Number of } \\
\text { entries (\%) }\end{array}$ \\
\hline Bone metastasis-free survival & bone MFS & $8(3.43)$ \\
\hline $\begin{array}{l}\text { Biochemical progression/ } \\
\text { recurrence-free survival }\end{array}$ & BPFS & $104(44.64)$ \\
\hline Clinical failure-free survival & CFFS & $7(3.00)$ \\
\hline $\begin{array}{l}\text { Castration resistant prostate } \\
\text { cancer-free survival }\end{array}$ & CRPC FS & $6(2.58)$ \\
\hline Cancer-specific survival & CSS & $6(2.58)$ \\
\hline Disease-free survival & DFS & $11(4.72)$ \\
\hline Disease-specific survival & DSS & $2(0.86)$ \\
\hline Metastasis-free survival & MFS & $6(2.58)$ \\
\hline Overall suvival & OS & $47(20.17)$ \\
\hline Progression-free survival & PFS & $5(2.17)$ \\
\hline Percentage survival & PS & $13(5.58)$ \\
\hline $\begin{array}{l}\text { Recurrence/relapse-free } \\
\text { survival }\end{array}$ & RFS & $18(7.73)$ \\
\hline
\end{tabular}

Twelve different endpoints were considered. After verifying endpoint definitions in respective studies, endpoints with redundant meanings were categorised into the same group. If the studies did not provide definitions or the definitions were different between studies, endpoint with redundant meanings were not categorised together.

Within the eight signatures, only miRs let-7a-5p and miR-223 were present in multiple miR signatures. In Mihelich et al., both were grouped into a panel with five other miRs and their expression levels were significantly downregulated in recurrent patients compared to non-recurrent patients [56]. In Nam et al. miR-223, and in Fredsoe et al. let-7a-5p, were grouped into signature panels for their predictive power to significantly distinguish between recurrent and non-recurrent $\mathrm{PCa}$ cases $[57,58]$. Interestingly, although prognostic as part of miR signatures, neither let-7a-5p nor miR-223 have been reported as individually prognostic predictors. However, 16 out of the 36 unique miRs in the signature panels (miR-10b-5p, -130b-3p, -139$5 p,-145-5 p,-17-5 p,-19 a-3 p,-200 b-3 p,-20 a-5 p,-221-3 p,-23 a-3 p$, $-301 a 3 p,-326,-374 b-5 p,-375,-652-3 p$ and $-96-5 p)$ were reported as individually prognostic in multiple studies (Supplementary Table S1). For 11 out of these 16 (miR-10b-5p, -130b-3p, -145-5p, $-17-5 p,-19 a-3 p,-23 a-3 p,-301 a-3 p,-326,-374 b-5 p,-652-3 p$ and $-96-5 p)$, their individual association with progression in corresponding studies was consistent with the direction of expression in signature panel studies.

\section{Identification of miR biomarkers for prostate cancer recurrence following radical prostatectomy: a systematic reanalysis of publicly available $\mathbf{m i R}$ profile data}

Study selection and sample characteristics of eligible datasets. A total of 185 studies were retrieved from the initial literature search. After title and abstract screening, 164 ineligible articles such as metaanalyses, reviews and studies based on non-tissue datasets or nonPCa studies were removed. Full-text screening removed a further 16 studies as their datasets were not publicly available $(n=7)$, did not have follow-up information $(n=5)$, could not share clinical information due to patient confidentiality $(n=2)$, contained inconsistent clinical information $(n=1)$ or were categorised as duplicate due to using public datasets already included in this reanalysis $(n=$ 1). Ultimately, five studies, containing six datasets, were eligible for the reanalysis. The workflow for the selection of studies is detailed in Fig. $1 \mathrm{~b}$ and study characteristics are reported in Table 1.

MiRs were profiled from tissue samples collected from men who underwent RP in all datasets. The endpoint for the datasets was BCR, which was defined by the majority of the datasets as a rise in serum PSA levels $\geq 0.2 \mathrm{ng} / \mathrm{ml}$ on two or more occasions, consistent with the European Association of Urology guidelines [59]. Only GSE36738 did not specify the number of rising PSA measurements required to classify a BCR event. The majority of the datasets contained accompanying clinical variables: age at diagnosis, PSA at diagnosis, Gleason score and tumour stage. Only GSE88958 did not contain tumour stage information. The sample characteristics for these studies are provided in Supplementary Table S5.

Association of clinicopathological features with disease relapse. The associations of clinicopathological features (age, serum PSA at diagnosis, Gleason score sum and tumour stage) with disease relapse were tested in each dataset, and a REM meta-analysis model was employed to summarise the overall effect across the datasets (Supplementary Fig. S3). Although non-significant, higher age and PSA levels at diagnosis associated with a higher risk of BCR (Supplementary Fig. S3a, S3b). Higher Gleason score sum $(\geq 8)$ and higher tumour stages (T3+T4) had a significant and stronger association with BCR (pooled HR >3; Supplementary Fig. S3c, S3d). Gleason score sum, tumour stage and PSA at diagnosis are the standard prognostic features as per the National Institute for Healthcare and Excellence and European Association of Urology guidelines $[12,13]$. Thus, the multivariate models testing the association of miR expression with BCR were adjusted for these three confounding variables.

MiRs that consistently associate with disease relapse: a univariate analysis. Univariate Cox $\mathrm{PH}$ regression followed by a REM metaanalysis was performed for 162 miRs that were common in all six datasets. Pooled HR estimates for $18 \mathrm{miRs}$ were significantly associated with BCR (Table 4, Supplementary Fig. S4). Of these, 17 miRs (let-7a-5p, miR-125b-5p, -133a-3p, -135a-5p, -148a-3p, -155$5 p,-203 a-3 p,-204-5 p,-218-5 p,-222-3 p,-26 b-5 p,-30 a-3 p,-30 c-5 p$, $-30 e-3 p,-374 a-5 p,-455-5 p$ and miR-582-5p) had negative association, while only miR-425-3p had positive association with $B C R$. The Q-test for heterogeneity was not significant for any of the miRs and $\mathrm{I}^{2}$ statistic ranged from $0-40 \%$, suggesting moderate levels of heterogeneity between the datasets.

MiRs that consistently associate with disease relapse: a multivariate analysis. A total of $164 \mathrm{miRs}$ were common between the five datasets considered for the multivariate analysis.

The analysis revealed only 16 miRs significantly associated with BCR (Table 4, Supplementary Fig. S5). 13 miRs (let-7a-5p, miR-1-3p, $-148 a-3 p,-203 a-3 p,-20 a-5 p,-221-3 p,-26 b-5 p,-30 a-3 p,-30 c-5 p$, $-30 e-3 p,-30 e-5 p,-374 a-5 p$ and $-582-5 p)$ had negative association and three miRs (miR-130b-3p, $-181 b-5 p$ and $-425-3 p$ ) had positive association with disease relapse. The Q-tests for heterogeneity for these miRs were non-significant and the $\mathrm{I}^{2}$ value ranged from 0 to $30 \%$. These values represent moderate to no heterogeneity between the datasets. Overall, ten miRs (let-7a-5p, miR-148a-3p, $-203 a-3 p,-26 b 5 p,-30 a-3 p,-30 c-5 p,-30 e-3 p,-374 a-5 p,-425-3 p$ and $-582-5 p)$ were significantly prognostic in both univariate and multivariate meta-analyses (Table 4). The consistent trend in association with relapse of these $10 \mathrm{miRs}$, despite clinical and methodological differences between the datasets and even after being adjusted for confounding clinicopathological features, demonstrates replicability and robustness, and supports these miRs as ideal candidates for further investigation as prognostic PCa biomarkers.

\section{MiRs with consistent association with prostate cancer progression: agreement between systematic review and data reanalysis}

In the systematic review, five miRs, let-7b-5p, miR-145-5p, miR152-3p, miR-195-5p and miR-224-5p, were identified as consistently individually prognostic, of which the latter four miRs were 


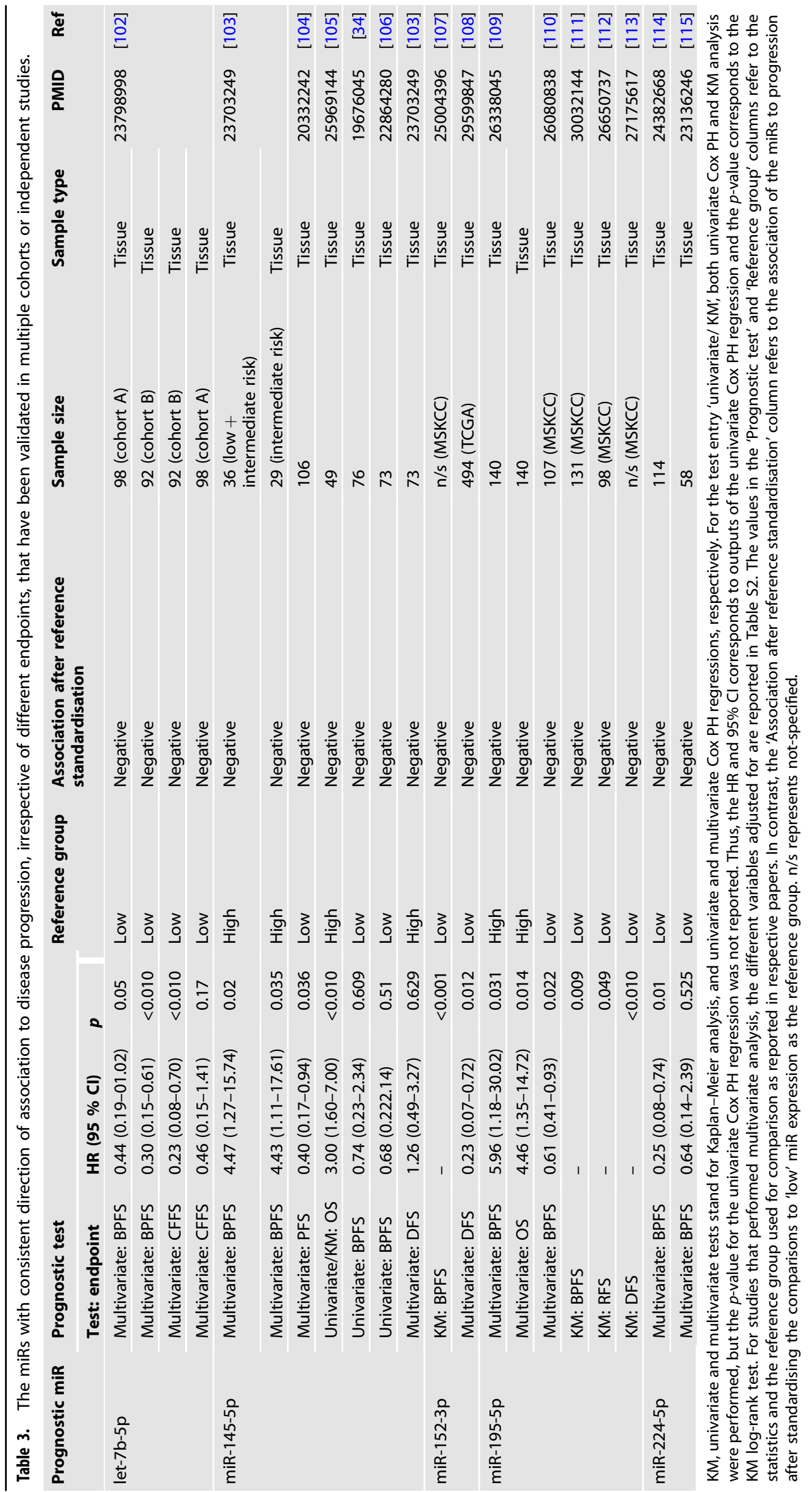




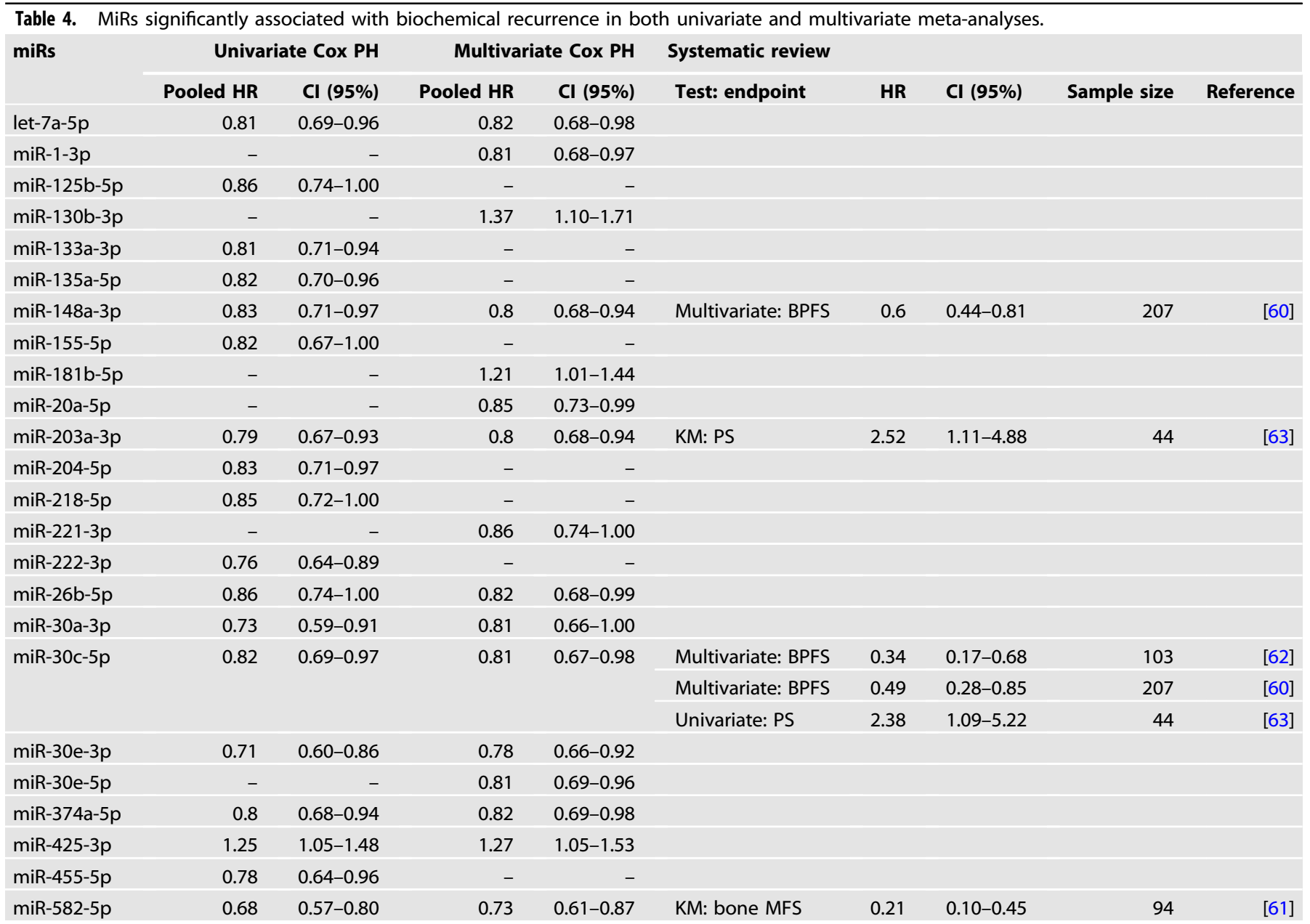

A set of 18 and 16 miRs were significant in the univariate and multivariate analyses, respectively. Ten miRs were significant in both analyses. Four miRs (miR148a-3p, miR-203a-3p, miR-30c-5p and miR-582-5p) out of these ten have been identified as prognostic in independent publications, although the direction of association with progression is not consistent for miR-203a-3p and miR-30c-5p between my findings and the independent publications. KM, univariate and multivariate tests refer to Kaplan-Meier analysis, univariate $\mathrm{Cox}$ PH regression and multivariate $\mathrm{Cox} \mathrm{PH}$ regression respectively. In the multivariate Cox $\mathrm{PH}$, the adjusted variables were Gleason score, tumour stage, and PSA. A total of five and six datasets were included in the univariate and multivariate meta-analyses, respectively. KM Kaplan-Meier. For the full form of the abbreviated endpoints, refer to Table 2.

evaluated in the multivariate meta-analysis. However, the association of these four miRs with BCR were non-significant and inconsistent in the data reanalysis (Supplementary Fig. S6).

In the reanalysis overall, ten miRs, let-7a-5p, miR-148a-3p, miR203a-3p, miR-26b-5p, miR-30a-3p, miR-30c-5p, miR-30e-3p, miR$374 a-5 p$, miR-425-3p and miR-582-5p, were validated as significantly prognostic of BCR post-RP. Among these, only four miRs (miR-148a-3p, miR-582-5p, miR-30c-5p and miR-203a-3p) were identified as individually prognostic in the systematic review (Table 4). The direction of association of miR-148a-3p and miR$582-5 p$ with progression endpoints BPFS and bone metastasis-free survival, respectively, in the review were consistent with the direction of association of the miRs with $B C R$ in the reanalysis $[60,61]$. MiR-30c-5p was reported as prognostic in three independent studies; Ling et al. and Zhao et al. reported negative association of miR-30c-5p expression with BPFS, which were consistent with the results from the meta-analysis $[60,62]$. However, the findings of Huang et al. were inconsistent as they reported positive association of miR-30c expression with $\mathrm{PCa}$ patient survival [63]. For miR-203a-3p, its direction of association with survival also conflicted with the findings of the reanalysis [63]. The inconsistencies for miR-30c-5p and miR-203a-3p could potentially be due to differences in endpoints or statistical approaches, such as inclusion of different confounder variables in the multivariate models. Although there were no overlaps between miRs identified as of interest in the systematic review and reanalysis, two miRs: miR-148a-3p and miR-582-5p (Fig. 2, Table 4), were identified as consistently predictive of BCR in the reanalysis and had at least one publication in the systematic review verifying their association $[60,61]$. Therefore, these two miRs are ideal candidates to follow-up as individual prognostic markers for PCa.

\section{DISCUSSION}

In this work, we addressed the problem of inconsistent and conflicting reports of prognostic miRs in $\mathrm{PCa}$ in the literature by undertaking a systematic review that summarised and identified consistently reported prognostic miR biomarkers in localised PCa. We next performed a systematic reanalysis of six publicly available miR profile datasets, which identified tumour tissue-derived miRs consistently associated with BCR in post RP samples. Two miRs miR-148a-3p and miR-582-5p were validated as independently prognostic of $\mathrm{PCa}$ progression in the review and reanalysis, despite significant heterogeneity between studies, and thus present as promising prognostic biomarkers for PCa progression. 
a

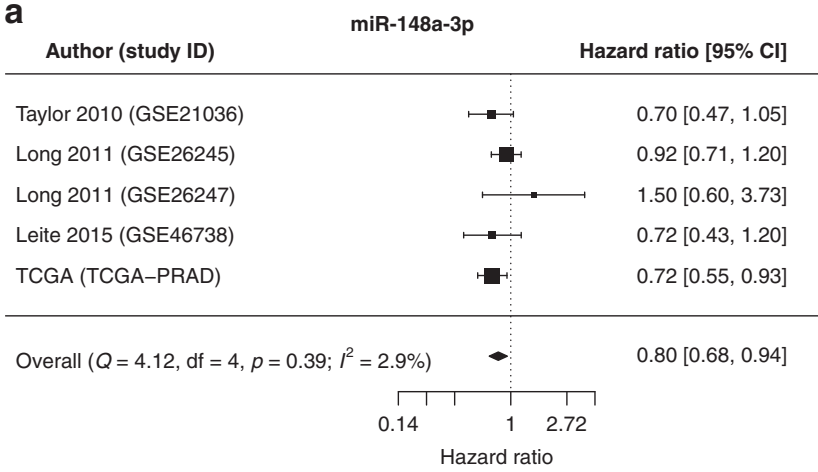

b

\begin{tabular}{llr}
\multicolumn{1}{c}{ Author (study ID) } & HiR-582-5p & Hazard ratio [95\% Cl] \\
\hline Taylor 2010 (GSE21036) & $0.58[0.38,0.89]$ \\
Long 2011 (GSE26245) & & $0.83[0.57,1.19]$ \\
Long 2011 (GSE26247) & & $0.71[0.41,1.25]$ \\
Leite 2015 (GSE46738) & & $0.33[0.13,0.83]$ \\
TCGA (TCGA-PRAD) & & $0.80[0.61,1.04]$
\end{tabular}

Overall $\left(Q=4.86, \mathrm{df}=4, p=0.30 ; l^{2}=0.0 \%\right)$ $-$ $0.73[0.61,0.87]$

\begin{tabular}{l|l|l|}
\hline 0.14 & 1 & 2.72 \\
\hline & Hazard ratio
\end{tabular}

Fig. 2 Association of miR-148a-3p and miR-582-5p with biochemical relapse (BCR). MiR-148a-3p (a) and miR-582-5p (b) expression levels show significant association to $B C R$ in the multivariate metaanalysis.

\section{miR-148a-3p}

MiR-148a-3p is one of the most commonly dysregulated miRs in human cancers. Its downregulation has been observed in various cancers such as bladder, oesophageal, gastric, breast, colorectal and ovarian cancers [64-70]. Upregulation of miR-148a has also been detected in osteocarcinoma and glioblastoma [71, 72]. In $\mathrm{PCa}$, upregulation of miR-148a-3p levels has been shown in prostate tumour tissue in comparison to adjacent normal tissue [33]. Upregulation was also observed in serum and urine of PCa patients in comparison to healthy controls $[40,73]$. In contrast, levels of miR-148a-3p have been reported as lower in CRPC cell lines PC3 and DU145 compared to lines representing therapyresponsive disease $[74,75]$. Similarly, in PCa patients, downregulation of the miR has been reported in CRPC cases compared to $\mathrm{BPH}$ cases and in high-grade tumours compared to low-grade tumours [32, 76].

Although expression of miR-148a-3p is variably reported in the literature, studies investigating its biological role in PCa generally suggest a tumour suppressive role. Sengupta et al. showed downregulation of miR-148a-3p in CRPC and identified DNA methylatransferase $D N M T 1$, a gene upregulated in several cancers, as a target of the miR [75]. They reported that the two molecules exhibit a negative loop in PCa: while DNMT1 enzyme methylates the miR promoter and silences miR expression, miR148a directly targets DNMT1, whose repression leads to induction of apoptosis and repression of cell proliferation and migration. They also demonstrated that ectopic expression of miR-148a-3p repressed anti-apoptotic $B C L 2$ in PC3 cells promoting apoptosis. Suppression of DNMT1 by miR-148-3p has been reported in pancreatic, liver, bladder, oesophageal and gastric cancers [64-67, 77, 78]. Targeting of $B C L 2$ by miR-148a-3p has also been reported in colorectal and pancreatic cancers $[69,79]$. Additionally, a study by Fujita et al. showed miR-148a-3p expression increased chemosensitivity in PC3 cells by directly targeting mitogen-and stress-activated protein kinase, MSK1 [74]. These studies demonstrate that miR$148 a-3 p$ plays a role in promoting an anti-survival, tumour suppressive phenotype via similar mechanisms in various cancers including PCa and its loss is not only a good indicator of tumour progression but also shows potential to serve as a biomarker for therapeutic response in PCa.

\section{miR-582-5p}

Similar to miR-148a-3p, miR-582-5p is reported to act as both an oncogene and a tumour suppressor in various cancers. In gastric, bladder, non-small cell lung cancers and endometrial carcinoma, miR582-5p levels are downregulated and shown to suppress proliferation, migration, invasion and promote apoptosis [80-83]. Conversely, in colorectal cancer and pituitary adenomas, it is overexpressed and promotes proliferation [84, 85]. The clinical significance of miR-582-5p in PCa is not yet elucidated and the literature presents conflicting evidence. The most recent research on miR-582-5p in PCa investigated its role in promoting bone metastasis; lower miR-582-5p expression was reported in $\mathrm{PCa}$ tissues with bone metastasis compared to PCa tissues without bone metastasis [61]. The study reported that lower miR-582-5p expression was significantly associated with shorter bone metastasis-free survival. They also demonstrated that overexpression of the miR in mice bearing PC3 tumour xenografts repressed bone metastasis and over-expression in PCa cell lines PC3, VCaP and C42B repressed cell invasion and migration. Mechanistically, the study proposed that miR-582-3p exerted its anti-invasion and migration properties by directly inhibiting components of the TGF $\beta$ signalling pathway (SMAD2, TGFBRI and TGFBRII) and subsequently the pathway itself. In a separate study, Maeno et al. developed an AR-positive, androgenindependent xenograft model KUCaP2 and cell line AlLNCaP\#1, and observed upregulation of miR-582-5p in these models in comparison to their androgen dependent counterparts [86]. They also demonstrated that suppression of the miR decreased cell proliferation in AILNCaP\#1, suggesting an oncomiRic role of miR582-5p in the transition of PCa from hormonesensitive to more aggressive castration-resistant phenotypes. These limited studies on miR-582-3p report conflicting roles in tumour progression, which indicate a dual role of the miR at different stages of progression from invasion and metastasis to the bone, to transition from androgen-dependent to aggressive CRPC. Given the identification of $\mathrm{miR}-582-5 \mathrm{p}$ as a potential prognostic candidate for $\mathrm{PCa}$, further research into its exact role in $\mathrm{PCa}$ tumour progression is warranted.

\section{Limitations}

One of the major issues highlighted by this study is the inconsistent findings between studies and datasets despite their common aim to identify prognostic miR biomarkers in PCa. These inconsistencies are mainly due to clinical and methodological heterogeneities that can arise at many points during the study. Due to the nature of retrospective cohort studies, clinical heterogeneity which encompasses factors such as race, family history, co-morbidity, treatment history, time to outcome and loss of follow up, was unavoidable. In the systematic review, a potential contributor to clinical heterogeneity was outcome endpoints. There were 12 different endpoints included in the systematic review as surrogates of disease progression. Further, many of the studies did not provide endpoint definitions. For studies that considered the same endpoints and provided endpoint definitions, definition heterogeneity still existed. This is evident in studies by Hulf et al. [87] and Nordby et al. [88] oth of which examined the association of miR-205 with BPFS but used different criteria to define BPFS. To minimise clinical heterogeneity in the reanalysis, we only used studies examining association of miRs with endpoint BPFS and samples originating from tumour 
tissues of patients who underwent RP and no other curative treatment.

It should be noted that BPFS and other PSA-based endpoints may not be ideal surrogate endpoints for disease progression. The ICECaP study, a large meta-analysis that aimed to determine clinically relevant endpoints for localised $\mathrm{PCa}$, determined metastasis-free survival (MFS) as the most appropriate surrogate for PCa specific survival $[89,90]$. However, BCR has been shown to be associated with increased risk of PCa metastasis and cancerspecific mortality and further is the most common endpoint recorded and reported in publications [7-11]. This is evident in the systematic review where almost half the studies (44\%) considered BPFS, while only $6 \%$ of studies considered bone-/metastasis free survival, as endpoints. Moving forward, studies should consider evidence-based clinically relevant endpoints for studies focusing on disease progression. Similar to miR expression profile after RP, miR expression profiles also change at recurrence after radical radiotherapy, another curative treatment option for primary PCa. Several studies have identified miRs that change in expression in response to radiation and miRs that are involved in regulation of radiosensitivity in $\mathrm{PCa}$ [91-95]. It would be interesting to investigate and compare miR expression profile at recurrence after different curative treatments and their association with MFS [96-98].

Methodological heterogeneity, due to differences in study design, sample preparation methods, sample types, profiling technologies and threshold values for a positive result, was also present in the analyses. Besides these factors, one of the sources of methodological heterogeneity that may have influenced results were the different statistical tests (KM analysis or Cox $\mathrm{PH}$ regression) performed by different studies. The KM analysis only allows categorical variables as predictors, which can lead to weakening or loss of potential signal. It also cannot adjust to multiple predictors. Cox $\mathrm{PH}$ regression, on the other hand, is more flexible and allows for both categorical and continuous variables as predictors. Multiple predictors can also be added into a $\mathrm{Cox} \mathrm{PH}$ model, allowing for adjustment of confounding variables. For this reason, when a study in the systematic review reported outcomes of both $\mathrm{Cox} \mathrm{PH}$ and $\mathrm{KM}$ analyses, only the Cox $\mathrm{PH}$ results were extracted. However, even with adjustment for confounders in the Cox $\mathrm{PH}$ regression, there was potential for further heterogeneity to be introduced as different studies adjusted for different confounders. For example, Amankwah et al. [99], MelboJorgensen et al. [100] and Guan et al. [101] examined the association of miR21-5p with progression using a multivariate $\mathrm{Cox} \mathrm{PH}$ model, but each study considered different confounders in their model (Supplementary Table S2). Although appropriate measures were taken to reduce heterogeneity, it cannot be completely eliminated. This highlights the need for standardization of methodology and protocols in the field of biomarker discovery in order to derive more accurate conclusions from future investigations.

Overall, the minimisation of heterogeneity will require cannot be completely eliminated. This calls for the need for standardization of methodology and protocols at the pre-analytical level (such as sample collection, storage, profiling techniques and reagents used) and at the post-analytical level (such as data normalisation, processing tools/ pipelines and statistical analyses) in order to reduce as much technical variability as possible and facilitate more accurate conclusions from future investigations. Prospective studies with well- and pre-defined experimental design and research questions need to be devised to address clinical heterogeneity.

Besides heterogeneity, another major limitation in the systematic reanalysis was the limited number of publicly available datasets. Numerous studies generate novel miR expression data, but most do not make their data publicly available. This led to the inclusion of only six datasets for the systematic reanalysis. Additionally, the studies included in the reanalysis had a class imbalance problem whereby the proportion of samples that experienced the outcome were disproportionately lower than the samples that did not (Table S5). Insufficient datasets and class imbalance is a major problem of working with biomedical data, reducing the power of the study and potentially leading to biased conclusions specific to the cohorts in the analyses rather than the general population. Our research prompts for a more transparent system whereby researchers make their datasets available to other researchers. This will not only be useful for providing external validation cohorts but will also allow for scrutiny of the methodologies and analyses employed, which will improve scientific rigour and expedite biomarker discovery research.

The bottleneck in miR biomarker discovery seems to be the analytical and clinical validation step; this is highlighted in our results where we identified methodological heterogeneity between studies and lack of clinical validation in independent studies. Given the requirement for high quality and high-volume data, these are major challenges hindering progress in the field; addressing them is paramount for expediting miR biomarker research and successful translation of miR markers from the bench to the clinic.

\section{CONCLUSION}

This is the first systematic review and only the second metaanalysis of miR profile data, updated and expanded with newer datasets and larger sample sizes compared to the first metaanalysis performed in 2017 [43], to focus on prognostic miR markers in PCa. It reveals considerable research undertaken in the field of biomarker discovery in PCa and reports all credible prognostic miRs reported so far. These findings present a valuable reference point for future studies and will be useful for regrouping strategies in investigations into prognostic miR biomarker research. This investigation also highlighted the lack of validation or inconsistent evidence for miRs frequently suggested to have prognostic biomarker potential. Only miR-148a-3p and miR-582$5 p$ were consistently associated with disease progression in multiple publications and datasets, indicating reliability in predicting prognosis. Nevertheless, their biological significance in PCa progression is still uncertain. Further research to verify the biological roles of these miRs is warranted to support investigations into their performance as prognostic PCa biomarkers.

\section{DATA AVAILABILITY}

All data analysed in the study are publicly available, no new data were generated.

\section{REFERENCES}

1. Prostate cancer statistics. 2020; https://www.cancerresearchuk.org/healthprofessional/cancer-statistics/statistics-by-cancer-type/prostate-cancer.

2. Gosselaar C, Roobol MJ, Schr"oder FH. Prevalence and characteristics of screendetected prostate carcinomas at low prostate-specific antigen levels: Aggressive or insignificant?. BJU Int. 2005;95:231-7. https://doi.org/10.1111/j.1464410X.2005.05324.x

3. Draisma G, Etzioni R, Tsodikov A, Mariotto A, Wever E, Gulati R, et al. Lead Time and Overdiagnosis in Prostate-Specific Antigen Screening : Importance of Methods and Context. J Natl Cancer Inst. 2009;101:374-83. https://doi.org/ 10.1093/jnci/djp001.

4. Prensner JR, Rubin MA, Wei JT, Chinnaiyan AM. Beyond PSA: the next generation of prostate cancer biomarkers. Sci Transl Med. 2012;4:127rv3-127rv3. https:// doi.org/10.1126/scitranslmed.3003180.

5. Amling CL, Blute ML, Bergstralh EJ, Seay TM, Slezak J, Zincke H. Long-term hazard of progression after radical prostatectomy for clinically localized prostate cancer: Continued risk of biochemical failure after 5 years. J Urol. 2000;164:101-5. https://doi.org/10.1016/S0022-5347(05)67457-5.

6. Han M, Partin AW, Zahurak M, Piantadosi S, Epstein J, Walsh PC. Biochemical (prostate specific antigen) recurrence probability following radical prostatectomy for clinically localized prostate cancer. J Urol. 2003;169:517-23. https://doi.org/ 10.1016/S0022-5347(05)63946-8. 
7. Briganti A, Karnes RJ, Gandaglia G, Spahn M, Gontero P, Tosco L, et al. Natural history of surgically treated high-risk prostate cancer. Urologic Oncol: Semin Original Investig. 2015;33:163.e7-163.e13. https://doi.org/10.1016/j.urolonc.2014.11.018.

8. Kim DK, Koo KC, Lee KS, Hah YS, Rha KH, Hong SJ, et al. Time to disease recurrence is a predictor of metastasis and mortality in patients with High-risk prostate cancer who achieved undetectable prostate-specific Antigen Following robot-assisted radical prostatectomy. J Korean Med Sci. 2018;33:1-10. https:// doi.org/10.3346/jkms.2018.33.e285.

9. Pound CR, Partin AW, Eisenberger MA, Chan DW, Pearson JD, Walsh PC. Natural history of progression after PSA elevation following radical prostatectomy. J Am Med Assoc. 1999;281:1591-7. https://doi.org/10.1001/jama.281.17.1591.

10. Freedland SJ, Humphreys EB, Mangold LA, Eisenberger M, Dorey FJ, Walsh PC, et al. Risk of prostate cancer-specific mortality. JAMA. 2005;294:433-9.

11. van den Broeck T, van den Bergh RCN, Arfi N, Gross T, Moris Lisa, Briers E, et al. Prognostic value of biochemical recurrence following treatment with curative intent for prostate cancer: a systematic review. Eur Urol. 2019;75:967-87. https:// doi.org/10.1016/j.eururo.2018.10.011.

12. Mottet N, Bellmunt J, Bolla M, Briers E, Cumberbatch MG, Santis MD, et al. EAUESTRO-SIOG guidelines on prostate cancer. Part 1: screening, diagnosis, and local treatment with curative intent. Eur Urol. 2017;71:618-29. https://doi.org/ 10.1016/j.eururo.2016.08.003.

13. NICE. Prostate cancer diagnosis and management. Lancet. 2019; https://doi.org/ 10.1016/S0140-6736(96)07393-X.

14. Serefoglu EC, Altinova S, Ugras NS, Akincioglu E, Asil E \& Balbay MD. How reliable is 12 -core prostate biopsy procedure in the detection of prostate cancer? Can Urol Assoc J. 2013;7:E293-8.

15. Ahmed HU, El-Shater Bosaily A, Brown LC, Gabe R, Kaplan R, Parmar MK, et al. Diagnostic accuracy of multi-parametric MRI and TRUS biopsy in prostate cancer (PROMIS): a paired validating confirmatory study. Lancet. 2017;389:815-22. https://doi.org/10.1016/S0140-6736(16)32401-1.

16. Klein EA, Cooperberg MR, Magi-Galluzzi C, Simko JP, Falzarano SM, Maddala T, et al. A 17-gene assay to predict prostate cancer aggressiveness in the context of gleason grade heterogeneity, tumor multifocality, and biopsy undersampling. Eur Urol. 2014;66:550-60. https://doi.org/10.1016/j.eururo.201405.004.

17. Cuzick J, Swanson GP, Fisher G, Brothman AR, Berney DM, Reid JE, et al. Prognostic value of an RNA expression signature derived from cell cycle proliferation genes in patients with prostate cancer: a retrospective study. Lancet Oncol. 2011;12:245-55. https://doi.org/10.1016/S1470-2045(10)70295-3.

18. N Erho, A Crisan, IA Vergara, AP Mitra, M Ghadessi, C Buerki, et al. Discovery and validation of a prostate cancer genomic classifier that predicts early metastasis following radical prostatectomy. PLoS ONE. 2013;8:e66855. https://doi.org/ 10.1371/journal.pone.0066855.

19. Cullen J, Rosner IL, Brand TC, Zhang N, Tsiatis AC, Moncur J, et al. A biopsy-based 17-gene genomic prostate score predicts recurrence after radical prostatectomy and adverse surgical pathology in a racially diverse population of men with clinically low- and intermediate-risk prostate cancer. Eur Urol. 2015;68:123-31. https://doi.org/10.1016/j.eururo.2014.11.030.

20. Brand TC, Zhang N, Crager MR, Maddala T, Dee A, Sesterhenn IA, et al. Patientspecific meta-analysis of 2 clinical validation studies to predict pathologic outcomes in prostate cancer using the 17-gene genomic prostate score. Urology. 2016;89:69-75. https://doi.org/10.1016/j.urology.2015.12.008.

21. Spratt DE, Zhang J, Santiago-Jimenez M, Dess RT, Davis JW, Den RB, et al. Development and validation of a novel integrated clinical-genomic risk group classification for localized prostate cancer. J Clin Oncol. 2018;36:581-90. https:// doi.org/10.1200/JCO.2017.74.2940.

22. Bartel. DP. Metazoan microRNAs. Cell. 2018;173:20-51. https://doi.org/10.1016/j. cell.2018.03.006

23. Gebert LFR, MacRae IJ. Regulation of microRNA function in animals. Nat Rev Mol Cell Biol. 2019;20:21-37. https://doi.org/10.1038/s41580-018-0045-7.

24. Volinia S, Calin GA, Liu C-G, Ambs S, Cimmino A, Petrocca F, et al. A microRNA expression signature of human solid tumors defines cancer gene targets. Proc Natl Acad Sci USA. 2005:103:2257-61.

25. Lu J, Getz G, Miska EA, Alvarez-saavedra E, Lamb J, Peck D, et al. MicroRNA expression profiles classify human cancers. Nature. 2005;435:834-8. https://doi. org/10.1038/nature03702.

26. Schwarzenbach $\mathrm{H}$, Nishida N, Calin GA, Pantel K. Clinical relevance of circulating cell-free microRNAs in cancer. Nat Rev Clin Oncol. 2014;11:145-56. https://doi. org/10.1038/nrclinonc.2014.5.

27. Koppers-lalic D, Hackenberg M, Menezes RD. Non-invasive prostate cancer detection by measuring miRNA variants (isomiRs) in urine extracellular vesicles. Oncotarget. 2016;7:22566-78.

28. Mitchell PS, Parkin RK, Kroh EM, Fritz BR, Wyman SK, Pogosova-Agadjanyan EL et al. Circulating microRNAs as stable blood-based markers for cancer detection. Proc Natl Acad Sci USA. 2008;105:10513-8. https://doi.org/10.1073/ pnas.0804549105.
29. Krol J, Loedige I, Filipowicz W. The widespread regulation of microRNA biogenesis, function and decay. Nat Rev Genet. 2010;11:597-610.

30. Brase JC, Haese A, Steuber T, Johannes M, Schlomm T, Fa M. Circulating miRNAs are correlated with tumor progression in prostate cancer. Int J Cancer. 2011;128:608-16. https://doi.org/10.1002/ijc.25376.

31. Gantier MP, Mccoy CE, Rusinova I, Saulep D, Wang D, Xu D, et al. Analysis of microRNA turnover in mammalian cells following Dicer1 ablation. Nucleic Acids Res. 2011;39:5692-703. https://doi.org/10.1093/nar/gkr148.

32. Porkka KP, Pfeiffer MJ, Waltering KK, Vessella RL, Tammela TL, Visakorpi T. MicroRNA Expression profiling in prostate cancer. Cancer Res. 2007;67:6130-6. https://doi.org/10.1158/0008-5472.CAN-07-0533.

33. Szczyrba J, L“oprich E, Wach S, Jung V, Unteregger G. The MicroRNA profile of prostate carcinoma obtained by deep sequencing. Mol Cancer Res. 2010;8:529-39. https://doi.org/10.1158/1541-7786.MCR-09-0443.

34. Schaefer A, Jung M, Mollenkopf HJ, Wagner I, Stephan C, Jentzmik F, et al. Diagnostic and prognostic implications of microRNA profiling in prostate carcinoma. Int J Cancer. 2010;126:1166-76. https://doi.org/10.1002/ijc.24827.

35. Wach S, Nolte E, Szczyrba J, Stohr R, Hartmann A, Ørntoft T, et al. MicroRNA profiles of prostate carcinoma detected by multiplatform microRNA screening. Int J Cancer. 2012;130:611-21. https://doi.org/10.1002/ijc.26064.

36. Ngoc CHN, Xie W, Yang M, Hsieh C-L, Drouin S, Lee G-SM, et al. Expression differences of circulating microRNAs in metastastic castration resistant prostate cancer and low-risk, localized prostate cancer. Prostate. 2013;73:346-54. https:// doi.org/10.1002/pros.22572.

37. Watahiki Y, Macfarlane RJ, Gleave ME, Crea F, Wang Y, Helgason CD, et al. Plasma miRNAs as biomarkers to identify patients with castrationresistant metastatic prostate cancer. Int J Mol Sci. 2013;14:7757-70. https://doi.org/ 10.3390/ijms14047757.

38. Selth LA, Townley SL, Bert AG, Stricker PD, Sutherland PD, Horvath LG, et al. Circulating microRNAs predict biochemical recurrence in prostate cancer patients. Br J Cancer. 2013;109:641-50. https://doi.org/10.1038/bjc.2013.369.

39. Kim Y-K. Extracellular microRNAs as biomarkers in human disease. Chonnam Med J. 2015;51:51. https://doi.org/10.4068/cmj.2015.51.2.51.

40. Stuopelyte K, Daniunaite K, Bakavicius A, Lazutka JR, Jankevicius F, Jarmalaite. S. The utility of urine-circulating miRNAs for detection of prostate cancer. $\mathrm{Br} J$ Cancer. 2016;115:707-15. https://doi.org/10.1038/bjc.2016.233.

41. Suer I, Guzel E, Karatas OF, Creighton CJ, Ittmann M \& Ozen M. MicroRNAs as prognostic markers in prostate cancer. Prostate 2018;79:265-71. https://doi.org/ 10.1002/pros.23731.

42. RJ Bryant, T Pawlowski, JWF Catto, G Marsden, RL Vessella, B Rhees, et al. Changes in circulating microRNA levels associated with prostate cancer. $\mathrm{Br} J$ Cancer 2012;106:768-74. https://doi.org/10.1038/bjc.2011.595.

43. Pashaei E, Pashaei E, Ahmady M, Ozen M, Aydin N. Metaanalysis of miRNA expression profiles for prostate cancer recurrence following radical prostatectomy. PLoS ONE. 2017;12:1-23. https://doi.org/10.1371/journal. pone.0179543.

44. Liberati A, Altman DG, Tetzlaff J, Mulrow C, Gøtzsche PC, loannidis JP, et al. The PRISMA statement for reporting systematic reviews and metaanalyses of studies that evaluate healthcare interventions: explanation and elaboration. BMJ. 2009;339:b2700. https://doi.org/10.1136/bmj.b2700.

45. Viechtbauer W. Conducting meta-analyses in R with the metafor package. J Stat Softw. 2010;36:1-48.

46. mirbase: the microrna database. http://www.mirbase.org/.

47. Home - dbGaP - NCBI. https://www.ncbi.nlm.nih.gov/gap/.

48. GDC Data Transfer Tool. https://gdc.cancer.gov/access-data/gdc-data-transfertool.

49. Colaprico A, Silva TC, Olsen C, Garofano L, Cava C, Garolini D, et al. TCGAbiolinks: an R/Bioconductor package for integrative analysis of TCGA data. Nucleic Acids Res. 2016;44.8:e71. https://doi.org/10.1093/nar/gkv1507.

50. McCarthy DJ, Chen Y, Smyth GK. Differential expression analysis of multifactor RNA-Seq experiments with respect to biological variation. Nucleic Acids Res. 2012;40:4288-97. https://doi.org/10.1093/nar/gks042.

51. Home - GEO - NCBI. http://www.ncbi.nlm.nih.gov/geo/.

52. Long $Q$, Johnson $B A$, Osunkoya AO, Lai $Y-H$, Zhou W, Abramovitz $M$, et al. Protein-coding and MicroRNA biomarkers of recurrence of prostate cancer following radical prostatectomy. Am J Pathol. 2011;179:46-54. https://doi.org/ 10.1016/j.ajpath.2011.03.008.

53. Xu T, Su N, Liu L, Zhang J, Wang $H$, Zhang $W$ et al. miRBaseConverter: an R/ bioconductor package for converting and retrieving miRNA name, accession, sequence and family information in different versions of miRBase. BMC Bioinform. 2018;119(Suppl 19):514. https://doi.org/10.1186/s12859-018-2531-5.

54. Therneau TM, Grambsch PM. Modeling Survival Data: Extending the Cox Model. New York: Springer, 2000.

55. Feng S, Qian X, Li H, Zhang X. Combinations of elevated tissue miRNA-17-92 cluster expression and serum prostate-specific antigen as potential diagnostic 
biomarkers for prostate cancer. Oncol Lett. 2017;14:6943-9. https://doi.org/ 10.3892/ol.2017.7026

56. Mihelich BL, Maranville JC, Nolley R, Peehl DM, Nonn L. Elevated serum microRNA levels associate with absence of high-grade prostate cancer in a retrospective cohort. PLoS ONE. 2015;10:1-15. https://doi.org/10.1371/journal. pone. 0124245 .

57. Nam RK, Amemiya Y, Benatar T, Wallis CJD, StojcicBendavid J, Bacopulos S, et al. Identification and validation of a five MicroRNA signature predictive of prostate cancer recurrence and metastasis: a cohort study. J Cancer. 2015;6:1160-71. https://doi.org/10.7150/jca.13397.

58. Fredsøe J, Rasmussen AKI, Thomsen AR, Mouritzen P, Høyer S, Borre M, et al. Diagnostic and prognostic microRNA biomarkers for prostate cancer in cell-free urine. Eur Urol Focus. 2017;4:825-33. https://doi.org/10.1016/j.euf.2017.02.018.

59. Cornford P, Bellmunt J, Bolla M, Briers E, Santis MD, Gross T, et al. EAU-ESTROSIOG Guidelines on prostate cancer. Part II: treatment of relapsing, metastatic, and castration-resistant prostate cancer". Eur Urol. 2017;71:630-42. https://doi. org/10.1016/j.eururo.2016.08.002.

60. Zhao Z, Weickmann S, Jung M, Lein M, Kilic E, Stephan C, et al. A novel predictor tool of biochemical recurrence after radical prostatectomy based on a fivemicroRNA tissue signature. Cancers 2019;11:1603. https://doi.org/10.3390/ cancers 11101603

61. Huang S, Zou C, Tang Y, Wa Q, Peng X, Chen X, et al. miR-582-3p and miR-582$5 p$ suppress prostate cancer metastasis to bone by repressing tgf $\beta$ signaling. Mol Ther Nucleic Acids. 2019;16:91-104. https://doi.org/10.1016/j. omtn.2019.01.004.

62. Ling XH, Han ZD, Xia D, He HC, Jiang FN, Lin ZY, et al. MicroRNA-30c serves as an independent biochemical recurrence predictor and potential tumor suppressor for prostate cancer. Mol Biol Rep. 2014;41:2779-88. https://doi.org/10.1007/ s11033-014-3132-7.

63. Huang Z, Zhang L, Yi X, Yu X. Diagnostic and prognostic values of tissue hsamiR-30c and hsa-miR-203 in prostate carcinoma. Tumor Biol. 2016;37:4359-65. https://doi.org/10.1007/s13277-015-4262-9.

64. Wang X, Liang Z, Xu X, Li J, Zhu Y, Meng S, et al. MIR-148a-3p represses proliferation and EMT by establishing regulatory circuits between ERBB3/AKT2/ cmyc and DNMT1 in bladder cancer. Cell Death Dis. 2016;7:e2503-12. https:// doi.org/10.1038/cddis.2016.373.

65. ALombard AP, Mooso BA, Libertini SJ, Lim RM, Nakagawa RM, Vidallo KD, et al. miR-148a dependent apoptosis of bladder cancer cells is mediated in part by the epigenetic modifier DNMT1. Mol Carcinog. 2017;55:757-67. https://doi.org/ 10.1002/mc.22319.miR-148a.

66. Wang Y, Hu Y, Guo J, Wang L. MiR-148a-3p Suppresses the proliferation and invasion of esophageal cancer by targeting DNMT1. Genet Test Mol Biomark. 2019;23:98-104. https://doi.org/10.1089/gtmb.2018.0285.

67. Shi $H$, Chen X, Jiang $H$, Wang $X, Y u H$, Sun $P$, et al. miR-148a suppresses cell invasion and migration in gastric cancer by targeting DNA methyltransferase 1. Oncol Lett. 2018;15:4944-50. https://doi.org/10.3892/ol.2018.7907.

68. Yu J, Li Q, Xu Q, Liu L, Jiang B. MiR-148a inhibits angiogenesis by targeting ERBB3. J Biomed Res. 2011;25:170-7. https://doi.org/10.1016/S1674-8301(11) 60022-5.

69. Zhang H, Li Y, Huang Q, Ren X, Hu H, Sheng H, et al. MiR-148a promotes apoptosis by targeting $\mathrm{BCl}-2$ in colorectal cancer. Cell Death Differ. 2011;18:1702-10. https://doi.org/10.1038/cdd.2011.28.

70. Wang W, Dong J, Wang M, Yao S, Tian X, Cui X, et al. miR-148a-3p suppresses epithelial ovarian cancer progression primarily by targeting c-Met. Oncol Lett. 2018;15:6131-6. https://doi.org/10.3892/ol.2018.8110.

71. Ma W, Zhang X, Chai J, Chen P, Ren P, Gong M. Circulating miR-148a is a significant diagnostic and prognostic biomarker for patients with osteosarcoma. Tumor Biol. 2014;35:12467-72. https://doi.org/10.1007/s13277-0142565-x.

72. Kim J, Zhang Y, Skalski M, Hayes J, Kefas B, Schiff D, et al. MicroRNA-148a is a prognostic oncomiR that targets MIG6 and BIM to regulate EGFR and apoptosis in glioblastoma. Cancer Res. 2014;74:1541-53. https://doi.org/10.1158/00085472.CAN-13-1449.

73. Dybos SA, Flatberg A, Halgunset J, Viset T, Rolfseng T, Kvam S, et al. Increased levels of serum miR-148a-3p are associated with prostate cancer. Apmis. 2018;126:722-31. https://doi.org/10.1111/apm.12880.

74. Fujita Y, Kojima K, Ohhashi R, Hamada N, Nozawa Y, Kitamoto A, et al. MiR-148a attenuates paclitaxel resistance of hormone-refractory, drug-resistant prostate cancer PC3 cells by regulating MSK1 expression. J Biol Chem. 2010;285: 19076-84. https://doi.org/10.1074/jbc.M109.079525.

75. Sengupta $D$, Deb M, Patra SK. Antagonistic activities of miR148a and DNMT1: ectopic expression of miR-148a impairs DNMT1 mRNA and dwindle cell proliferation and survival. Gene. 2018;660:68-79. https://doi.org/10.1016/j. gene.2018.03.075.

76. Walter BA, Valera VA, Pinto PA, Merino. MJ. Comprehensive microRNA profiling of prostate cancer. J Cancer. 2013;4:350-7. https://doi.org/10.7150/jca.6394.
77. Long XR, He Y, Huang C, Li J. MicroRNA-148a is silenced by hypermethylation and interacts with DNA methyltransferase 1 in hepatocellular carcinogenesis. Int J Oncol. 2014;45:1915-22. https://doi.org/10.3892/ijo.2014.2373.

78. Zhan $\mathrm{Q}$, Fang $\mathrm{Y}$, Deng $\mathrm{X}$, Chen $\mathrm{H}$, Jin J, Lu X, et al. The interplay between miR148a and DNMT1 might be exploited for pancreatic cancer therapy. Cancer Investig. 2015;33:267-75. https://doi.org/10.3109/07357907.2015.1025794.

79. Zhang R, Li M, Zang W, Chen X, Wang Y, Li P, et al. MiR-148a regulates the growth and apoptosis in pancreatic cancer by targeting CCKBR and BCl-2. Tumor Biol. 2014;35:837-44. https://doi.org/10.1007/s13277-013-1115-2.

80. Uchino K, Takeshita F, Takahashi RU, Kosaka N, Fujiwara K, Naruoka H, et al. Therapeutic effects of microRNA-582-5p and $-3 p$ on the inhibition of bladder cancer progression. Mol Ther. 2013;21:610-9. https://doi.org/10.1038/mt.2012.269.

81. Jin Y, Tao LP, Yao SC, Huang QK, Chen ZF, Sun YJ, et al. MicroRNA-5825p suppressed gastric cancer cell proliferation via targeting AKT3. Eur Rev Med Pharmacol Sci. 2017;21:5112-20. https://doi.org/10.26355/eurrev_201711_13827.

82. Li L, Ma L. Upregulation of miR-582-5p regulates cell proliferation and apoptosis by targeting AKT3 in human endometrial carcinoma. Saudi J Biol Sci. 2018;25:965-70. https://doi.org/10.1016/j.sjbs.2018.03.007.

83. Wang LL, Zhang M. MiR-582-5p is a potential prognostic marker in human nonsmall cell lung cancer and functions as a tumor suppressor by targeting MAP3K2. Eur Rev Med Pharmacol Sci. 2018;22:7760-7. https://doi.org/10.26355/ eurrev-201811-16397.

84. Butz H, Likó I, Czirják S, Igaz P, Korbonits M, Rácz K, et al. MicroRNA profile indicates downregulation of the TGF $\beta$ pathway in sporadic non-functioning pituitary adenomas. Pituitary. 2011;14:112-24. https://doi.org/10.1007/s11102-010-0268-x.

85. Shu Z, Chen L, Ding D. miR-582-5P induces colorectal cancer cell proliferation by targeting adenomatous polyposis coli. World J Surgical Oncol. 2016;14:1-7. https://doi.org/10.1186/s12957-016-0984-4.

86. Maeno A, Terada Naoki, Uegaki M, Goto T, Okada Y, Kobayashi T, et al. Upregulation of miR-582-5p regulates cellular proliferation of prostate cancer cells under androgen-deprived conditions. Prostate. 2014;74:1604-12. https://doi. org/10.1002/pros.22877.

87. Hulf T, Sibbritt T, Wiklund ED, Patterson K, Song JZ, Stirzaker C, et al. Epigeneticinduced repression of microRNA-205 is associated with MED1 activation and a poorer prognosis in localized prostate cancer. Oncogene. 2013;32:2891-9. https://doi.org/10.1038/onc.2012.300.

88. Nordby $Y$, Richardsen E, Ness N, Donnem T, Patel HRH, Busund LT, et al. High miR-205 expression in normal epithelium is associated with biochemical failure An argument for epithelial crosstalk in prostate cancer?. Sci Rep. 2017;7:1-10. https://doi.org/10.1038/s41598-017-16556-2.

89. Xie W, Regan MM, Buyse M, Halabi S, Kantoff $P$, Sartor $O$, et al. Metastasis-free survival is a strong Surrogate of overall survival in localized prostate cancer. J Clin Oncol. 2017;35:3097-104. https://doi.org/10.1200/JCO.2017.73.9987.

90. Sweeney C, Nakabayashi M, Regan M, Xie W, Hayes J, Keating N, et al. The development of intermediate clinical endpoints in cancer of the prostate (ICECaP). J Natl Cancer Inst. 2015;107:djv261 https://doi.org/10.1093/jnci/djv261.

91. Morii A, Ogawa R, Watanabe A, Cui ZG, Takasaki I, Doi N, et al. Utilization of microRNAs with decreased expression levels in response to $X$-ray irradiation for fine-tuning radiation-controlled gene regulation. Int J Mol Med. 2013;32:9-16. https://doi.org/10.3892/ijmm.2013.1360.

92. Leung CM, Li SC, Chen TW, Ho MR, Hu LY, Liu WS, et al. Comprehensive microRNA profiling of prostate cancer cells after ionizing radiation treatment. Oncol Rep. 2014;31:1067-78. https://doi.org/10.3892/or.2014.2988.

93. Huang $X$, Taeb $S$, Jahangiri $S$, Emmenegger U, Tran E, Bruce J, et al. miRNA-95 mediates radioresistance in tumors by targeting the sphingolipid phosphatase SGPP1. Cancer Res. 2013;73:6972-86. https://doi.org/10.1158/0008-5472.CAN-13-1657.

94. Ray J, Haughey C, Hoey C, Jeon J, Murphy R, DuraPerez L, et al. miR-191 promotes radiation resistance of prostate cancer through interaction with RXRA. Cancer Lett. 2020;473:107-17. https://doi.org/10.1016/j.canlet.2019.12.025.

95. Hoey C, Ray J, Jeon J, Huang X, Taeb S, Ylanko J, et al. miRNA-106a and prostate cancer radioresistance: a novel role for LITAF in ATM regulation. Mol Oncol. 2018;12:1324-41. https://doi.org/10.1002/1878-0261.12328.

96. J Ni, J Bucci, L Chang, D Malouf, P Graham, Y Li. Targeting MicroRNAs in prostate cancer radiotherapy. Theranostics 2017;7: 3243-59. https://doi.org/10.7150/ thno.19934.

97. Lin H-M, Castillo L, Mahon KL, Chiam K, Lee BY, Nguyen Q, et al. Circulating microRNAs are associated with docetaxel chemotherapy outcome in castrationresistant prostate cancer. Br J Cancer. 2014;110:2462-71. https://doi.org/ 10.1038/bjc.2014.181.

98. Ottman R, Nguyen C, Lorch R, Chakrabarti R. MicroRNA expressions associated with progression of prostate cancer cells to antiandrogen therapy resistance. Mol Cancer. 2014;13:1-21. https://doi.org/10.1186/1476-4598-13-1.

99. Amankwah EK, Anegbe E, Park H, Pow-Sang J, Hakam A, Park JY. MiR-21, miR-221 and miR-222 expression and prostate cancer recurrence among obese and nonobese cases. Asian J Androl. 2013:15:226-30. https://doi.org/10.1038/aja.2012.160. 
100. Melbø-Jørgensen C, Ness N, Andersen S, Valkov A, Dønnem T, Al-Saad S et al. Stromal expression of miR-21 predicts biochemical failure in prostate cancer patients with Gleason score 6. PLoS ONE 2014;9:e113039. https://doi.org/ 10.1371/journal.pone.0113039.

101. Guan Y, Wu Y, Liu Y, Ni J, Nong S. Association of microRNA21 expression with clinicopathological characteristics and the risk of progression in advanced prostate cancer patients receiving androgen deprivation therapy. Prostate. 2016;76:986-93. https://doi.org/10.1002/pros.23187.

102. Schubert $M$, Spahn $M$, Kneitz S, Scholz CJ, Joniau S, Stroebel $P$, et al. Distinct microRNA expression profile in prostate cancer patients with early clinical failure and the impact of let-7 as prognostic marker in high-risk prostate cancer. PLoS ONE 2013;8:e65064. https://doi.org/10.1371/journal.pone.0065064.

103. Avgeris M, Stravodimos K, Fragoulis EG, Scorilas A. The loss of the tumoursuppressor miR-145 results in the shorter disease-free survival of prostate cancer patients. Br J Cancer. 2013;108.12:2573-581. https://doi.org/10.1038/bjc.2013.250.

104. Chen X, Gong J, Zeng $H$, Chen N, Huang R, Huang $Y$, et al. MicroRNA145 targets BNIP3 and suppresses prostate cancer progression. Cancer Res. 2010;70:2728-2738. https://doi.org/10.1158/0008-5472.CAN-09-3718.

105. Larne O, Hagman Z, Lilja H, Bjartell A, Edsjö A, Ceder Y. miR-145 suppress the androgen receptor in prostate cancer cells and correlates to prostate cancer prognosis. Carcinogenesis. 2015;36:858-66. https://doi.org/10.1093/carcin/bgv063.

106. Kang SG, Ha YR, Kim SJ, Kang SH, Park HS, Lee JG, et al. Do microRNA 96, 145 and 221 expressions really aid in the prognosis of prostate carcinoma?. Asian J Androl. 2012;14.5:752-57. https://doi.org/10.1038/aja.2012.68.

107. Theodore SC, Davis M, Zhao F, Wang H, Chen D, Rhim J, et al. MicroRNA profiling of novel African American and Caucasian Prostate Cancer cell lines reveals a reciprocal regulatory relationship of miR-152 and DNA methyltranferase 1 . Oncotarget. 2014;5:3512-25.

108. Ramalho-Carvalho J, Gonçalves CS, Graça I, Bidarra D, Pereira-Silva E, Salta S, et al. A multiplatform approach identifies miR-152-3p as a common epigenetically regulated onco-suppressor in prostate cancer targeting TMEM97. Clin Epigenetics. 2018;10:1-15. https://doi.org/10.1186/s13148-018-0475-2.

109. Guo J, Wang M, Liu X. MicroRNA-195 suppresses tumor cell proliferation and metastasis by directly targeting BCOX 1 in prostate carcinoma. J Exp Clin Cancer Res. 2015;34:1-8. https://doi.org/10.1186/s13046015-0209-7.

110. Cai C, Chen QB, Han ZD, Zhang YQ, He HC, Chen JH, et al. miR-195 inhibits tumor progression by targeting RPS6KB1 in human prostate cancer. Clin Cancer Res. 2015;21.21:4922-34. https://doi.org/10.1158/10780432.CCR-15-0217.

111. Tao Z, Xu S, Ruan H, Wang T, Song W, Qian L, et al. MiR-195/-16 family enhances radiotherapy via $\mathrm{T}$ cell activation in the tumor microenvironment by blocking the PD-L1 immune checkpoint. Cell Physiol Biochem. 2018;48:801-814. https:// doi.org/10.1159/000491909.

112. Liu C, Guan H, Wang Y, Chen M, Xu B, Zhang L, et al. MIR-195 inhibits emt by targeting FGF2 in prostate cancer cells. PLoS ONE. 2015;10:1-13. https://doi.org/ 10.1371/journal.pone.0144073.

113. Zhang $X$, Tao T, Liu C, Guan H, Huang $Y$, Xu B, et al. Downregulation of miR-195 promotes prostate cancer progression by targeting HMGA1. Oncol Rep. 2016;36.1:376-82. https://doi.org/10.3892/or.2016.4797.

114. Lin ZY, Huang YQ, Zhang YQ, Han ZD, He HC, Ling XH, et al. MicroRNA-224 inhibits progression of human prostate cancer by downregulating TRIB1. Int Cancer. 2014;135:541-50. https://doi.org/10.1002/ijc.28707.

115. Mavridis K, Stravodimos K, Scorilas A. Downregulation and prognostic performance of microRNA 224 expression in prostate cancer. Clin Chem 2013;59:261-69. https://doi.org/10.1373/clinchem.2012.191502.

116. Taylor BS, Schultz N, Hieronymus H. Integrative genomic profiling of human prostate cancer. Cancer Cell. 2010;18.1:11-22. https://doi.org/10.1016/j. ccr.2010.05.026.

117. Leite KRM, Reis ST, Viana N, Morais DR, Moura CM, Silva IA, et al. Controlling RECK miR21 promotes tumor cell invasion and is related to biochemica recurrence in prostate cancer. J Cancer. 2015;6:292-301. https://doi.org/ 10.7150/jca.11038.

118. Abeshouse A, Ahn J, Akbani R, Ally A, Amin S, Andry CD, et al. The molecular taxonomy of primary prostate cancer. Cell. 2015;163:1011-25. https://doi.org/ 10.1016/j.cell.2015.10.025.

\section{ACKNOWLEDGEMENTS}

We would like to thank Inbar Levi, Dr. Clare Eckold, Dr. Damien Leach, Prof. Lisa Horvath and Dr. James Flannagan for valuable discussions, and Prof. Katie Leite and Prof. Mustafa Ozen for providing additional information on their datasets.

\section{AUTHOR CONTRIBUTIONS}

SR conducted the systematic review, data reanalysis and wrote/ revised the manuscript. GV provided support with the Cox regression analysis. CB and HK conceived the study, supervised the project and reviewed/ revised the manuscript.

\section{FUNDING}

This work was supported by Prostate Cancer UK through a Movember Foundation Studentship (TLD-S15-005). We would also like to acknowledge support from the Cancer Research UK Imperial Centre.

\section{ETHICS APPROVAL AND CONSENT TO PARTICIPATE}

The relevant ethics and consent information for each study used in the analysis and review is included in the corresponding cited reference.

\section{CONSENT TO PUBLISH}

Not applicable.

\section{COMPETING INTERESTS}

The authors declare no competing interests.

\section{ADDITIONAL INFORMATION}

Supplementary information The online version contains supplementary material available at https://doi.org/10.1038/s41416-021-01677-3.

Correspondence and requests for materials should be addressed to Charlotte L. Bevan or Hector C. Keun.

Reprints and permission information is available at http://www.nature.com/ reprints

Publisher's note Springer Nature remains neutral with regard to jurisdictional claims in published maps and institutional affiliations.

Open Access This article is licensed under a Creative Commons Attribution 4.0 International License, which permits use, sharing, adaptation, distribution and reproduction in any medium or format, as long as you give appropriate credit to the original author(s) and the source, provide a link to the Creative Commons license, and indicate if changes were made. The images or other third party material in this article are included in the article's Creative Commons license, unless indicated otherwise in a credit line to the material. If material is not included in the article's Creative Commons license and your intended use is not permitted by statutory regulation or exceeds the permitted use, you will need to obtain permission directly from the copyright holder. To view a copy of this license, visit http://creativecommons. org/licenses/by/4.0/.

(c) The Author(s) 2022 